signaling. Therefore, inhibition of CD39 and/or CD73 holds exceptional advantages over $\mathrm{A} 2 \mathrm{aR}$ blockade as both, $\mathrm{A} 2 \mathrm{aR}$ dependent and A2aR independent effects of ATP degradation products are targeted simultaneously.

Disclosure Information J. Festag: A. Employment (full or parttime); Significant; Secarna Pharmaceuticals GmbH \& Co. KG. T. Thelemann: A. Employment (full or part-time); Significant; Secarna Pharmaceuticals GmbH \& Co. KG. M. Schell: A. Employment (full or part-time); Significant; Secarna Pharmaceuticals GmbH \& Co. KG. S. Raith: A. Employment (full or part-time); Significant; Secarna Pharmaceuticals GmbH \& Co. KG. S. Michel: A. Employment (full or part-time); Significant; Secarna Pharmaceuticals GmbH \& Co. KG. R. Klar: A. Employment (full or part-time); Significant; Secarna Pharmaceuticals GmbH \& Co. KG. F. Jaschinski: A. Employment (full or parttime); Significant; Secarna Pharmaceuticals GmbH \& Co. KG.

\section{P03.03 ORGANIZATION, FUNCTION AND GENE EXPRESSION OF TERTIARY LYMPHOID STRUCTURES IN PDAC RESEMBLES LYMPHOID FOLLICLES IN SECONDARY LYMPHOID ORGANS}

${ }^{1} \mathrm{M}$ Thelen, ${ }^{1} \mathrm{MA}$ García-Márquez* ${ }^{2} \mathrm{~T}$ Nestler, ${ }^{2} \mathrm{~S}$ Wagener-Ryczek, ${ }^{1} \mathrm{~J}$ Lehmann, ${ }^{1} \mathrm{E}$ Staib, ${ }^{3} \mathrm{~F}$ Popp, ${ }^{3} \mathrm{~F}$ Gebauer, ${ }^{2} \mathrm{P}$ Lohneis, ${ }^{2} \mathrm{M}$ Odenthal, ${ }^{2} \mathrm{~S}$ Merkelbach-Bruse, ${ }^{3} \mathrm{C}$ Bruns, ${ }^{1} \mathrm{~K}$ Wennhold, ${ }^{4} \mathrm{M}$ von Bergwelt-Baildon, ${ }^{1,3} \mathrm{HA}$ Schlößer. ${ }^{1}$ Center for Molecular Medicine Cologne, University Hospital of Cologne, Cologne, Germany; ${ }^{2}$ Institute for Pathology, University Hospital of Cologne, Cologne, Germany; ${ }^{3}$ Department of General, Visceral and Cancer Surgery, University Hospital of Cologne, Cologne, Germany; ${ }^{4}$ Department of Internal Medicine III, Ludwig Maximilian University Munich, Munich, Germany

\subsection{6/jitc-2020-ITOC7.43}

Background Secondary lymphoid organs (SLO) are involved in induction and enhancement of anti-tumor immune responses on different tumor entities. Recent evidence suggests that antitumor immune responses may also be induced or enhanced in the tumor microenvironment in so called tertiary lymphoid structures (TLS). It is assumed that TLS represent a hotspot for $\mathrm{T}$ cell priming, B cell activation, and differentiation, leading to cellular and humoral anti-tumor immune response.

Methods FFPE-slides of 120 primary pancreatic ductal adenocarcinoma (PDAC) patients were immunohistochemically (IHC) stained for CD20, CD3, CD8 and HLA-ABC to analyze spatial distribution of tumor-infiltrating lymphocytes. 5-color immunofluorescence staining was performed to further investigate structural components of TLS in comparison to lymphoid follicles in SLOs. Microscope-based laser microdissection and Nanostring-base RNA expression analysis were used to compare gene expression in PDAC, TLS, SLOs and normal pancreatic tissue.

Results TLS were frequently detected in PDAC and were mainly localized along the invasive tumor margin. In less than $10 \%$ of the cases TLS were infiltrating the tumors. Interestingly, $20 \%$ of the patients had no TLS. Results of TLS will be correlated with clinical parameters, Immunoscore and immune escape mechanisms. 5-color Immunofluorescence staining revealed similar organization and function of TLS and SLO. Finally, gene expression analyzed by Nanostring revealed largely overlapping expression patterns in TLS and SLO.

Conclusions The results clearly demonstrate close similarities between SLO and TLS in terms of composition, distribution and gene expression Patterns.

Disclosure Information M. Thelen: None. M.A. García-Márquez: None. T. Nestler: None. S. Wagener-Ryczek: None. J.
Lehmann: None. E. Staib: None. F. Popp: None. F. Gebauer: None. P. Lohneis: None. M. Odenthal: None. S. MerkelbachBruse: None. C. Bruns: None. K. Wennhold: None. M. von Bergwelt-Baildon: None. H.A. Schlößer: None.

\section{P03.04 APPLYING MULTISPECTRAL UNMIXING AND SPATIAL ANALYSES TO EXPLORE TUMOR HETEROGENEITY WITH A PRE-OPTIMIZED 7-COLOR IMMUNO-ONCOLOGY WORKFLOW}

V Goubert*. Akoya Biosciences, Malborough, MA, USA

\subsection{6/jitc-2020-ITOC7.44}

Background The tumor microenvironment hosts a myriad of cellular interactions that influence tumor biology and patient outcomes. Multiplex immunofluorescence (mIF) provides the ability to investigate a large number of these interactions in a single tissue section, and has been shown to outperform other testing modalities for predicting response to immunotherapies. ${ }^{1}$ Multispectral imaging (MSI) improves the capabilities of mIF by providing the ability to spectrally unmix fluorescence signals. This increases the number of markers that can be probed in the same scan and allows for separation of true immunofluorescence signals from tissue autofluorescence background. Here, we apply MSI to explore spatial interactions observed in lung cancer samples using an end-to-end translational workflow based on the Phenoptics ${ }^{\mathrm{TM}}$ platform. The workflow includes a pre-optimized 7-color staining panel kit along with a pre-configured analysis algorithm for cell phenotyping. Using tissue microarrays (TMA), we demonstrate the heterogeneity of spatial interactions observed among different lung cancer samples and the improved sensitivity of detection afforded by unmixing multispectral scans.

Materials and Methods Formalin-fixed paraffin-embedded (FFPE) lung cancer TMA contained 120 cores $(1.5 \mathrm{~mm}$ diameter, US Biomax, Inc., Derwood, MD). The TMA was stained using the MOTiF ${ }^{\text {TM }}$ PD-1/PD-L1 Panel: Auto Lung Cancer Kit and pre-optimized protocol for the Leica BOND $\mathrm{RX}^{\mathrm{TM}}$. Whole slide 7-color MOTiF multispectral scan was acquired on Vectra Polaris ${ }^{\circledR}$ using pre-defined parameters. Phenochart ${ }^{\mathrm{TM}}$ software was used to identify cores for analysis. Scans were unmixed and analyzed with inForm ${ }^{\circledR}$ software using a pre-configured algorithm tailored to the MOTiF ${ }^{\mathrm{TM}}$ PD-1/PD-L1 Panel kit. With this algorithm, cells are assigned phenotypes using intensity thresholds for CD8, PD-1, FoxP3, CD68, and PanCK signal levels, subject to pre-defined marker priority rules. The rules limit co-positivity to any combination of CD8, FoxP3, and PD-1, but no combinations of those markers with CD68 or PanCK, and no combination of CD68 with PanCK. When threshold levels generate excluded combinations, priority is given to calls for CD8/FoxP3/PD-1 over CD68, which in turn has priority over PanCK. To explore the dynamic range of PD-L1, it was assessed via expression level (signal intensity), not phenotyping. Spatial analyses and visualizations were performed in $\mathrm{R}^{2}$ using the phenoptr and phenoptrReports packages $^{3}$, and custom scripts.

Results The pre-optimized Opal Polaris 7-Color PD-1/PD-L1 Lung Cancer Panel Kit was able to visualize the panel targets (PD-L1, PD-1, CD8, CD68, FoxP3, and Cytokeratin) across the variety of lung cancer samples in the TMA. Cell phenotyping and spatial analyses revealed core-to-core variations in cell densities and proximities among different markers. 
Measurement of the dynamic range of PD-L1 expression across different cores also revealed the improved sensitivity in PD-L1 detection provided by unmixing.

Conclusions The end-to-end Phenoptics staining, imaging, unmixing, and spatial analysis workflow described here provides a robust and sensitive platform for exploring the immune landscape within the tumor microenvironment.

Disclosure Information V. Goubert: None.

\section{P03.05 DEEP SPATIAL PROFILING OF THE IMMUNE LANDSCAPE OF MSI AND MSS COLORECTAL TUMORS}

SE Church, J Reeves, DR Zollinger, J McKay-Fleisch, AJ Bahrami, M Holpert*, AM White, MD Bailey, CR Merritt, M Hoang, S Warren, JM Beechem. NanString Technologies, Seattle, WA, USA

\subsection{6/jitc-2020-ITOC7.45}

Introduction In colorectal cancer (CRC) there have been many recent advances in immune related biomarkers that are both prognostic and predictive of response to immunotherapy. Microsatellite instability (MSI)/mismatch repair deficiency dMMR is present in $15-20 \%$ of CRCs and correlates with increased immunogenic mutations that often augment lymphocyte infiltration into the tumor microenvironment (TME). Additionally, location of tumor infiltrating $\mathrm{T}$ cells in two areas of the TME, the tumor center (CT) and invasive margin (IM) has also been shown to be prognostic and predictive of response to immunotherapy. Here we use multiplexed protein and RNA digital spatial profiling to elicit the immune landscape of MSI-MSS characterized CRC tumors.

Methods Forty-eight CRC tumors were analyzed for gene expression using the NanoString ${ }^{\circledR}$ nCounter ${ }^{\circledR}$ PanCancer IO $360^{\mathrm{TM}}$ Research Use Only (RUO) Gene Expression Panel and assessed for 48 cell typing and biological signatures, including MMR loss/MSI predictor and the Tumor Inflammation Signature (TIS). A subset of 18 CRC tumors (6 MSI-TIS-hi, 6 MSS-TIS-hi, 6 MSS-TIS-lo) was selected for analysis with the RUO GeoMx ${ }^{\mathrm{TM}}$ Digital Spatial Profiler (DSP) using 40 antibodies (human IO protein panel), or 84 RNA probes (human IO RNA panel). Selection of regions of interest (ROIs) in two locations, CT and IM were guided by staining with fluorescent markers (CD45, CD3, pan-CK, DNA). 300-600 $\mu \mathrm{M}$ diameter circle ROIs were selected, and in some cases segmented by pan-CK+/pan-CK-. For 2 immune hot samples contour profiling at the IM into stromal and tumor regions was performed using $1400+$ RNA probes with NGS readout.

Summary Using whole tissue gene expression analysis, we determined the TIS and IO 360 signature scores for 48 CRC tumors using PanCancer IO 360 assay. 18 tumors within this cohort were selected based on TIS status to further dissect the location-dependent immune contexture of the TME. Protein DSP confirmed loss of dMMR markers (MSH2/MLH1) and identified an increased amount of potentially suppressive macrophages (CD163+PD-L1+) in MSI-TIS-hi versus MSS-TIS-hi tumors. Segmentation of ROIs based on tumor versus stroma (pan-CK \pm ) identified samples with high proportions of tumorinvading TILs. Two MSI-TIS-hi profiled using probes against $1400+$ mRNA targets confirmed protein results (CD163 in IM) and identified tumor-related signatures corresponding to the inside of the tumor (Cytokeratins, HER2/ERBB2, MET).

Conclusions Here we show the use of novel high-plex spatial profiling to profile location and pathways in the TME of MSI and MSS CRC tumors. These findings elicit unique biology related to the location and signaling of immune cells, which have the potential to unveil targets for therapeutic combinations.

Disclosure Information S.E. Church: A. Employment (full or part-time); Significant; NanoString Technologies. J. Reeves: A. Employment (full or part-time); Significant; NanoString Technologies. D.R. Zollinger: A. Employment (full or parttime); Significant; NanoString Technologies. J. McKayFleisch: A. Employment (full or part-time); Significant; NanoString Technologies. A.J. Bahrami: A. Employment (full or part-time); Significant; NanoString Technologies. M. Holpert: A. Employment (full or part-time); Significant; NanoString Technologies. A.M. White: A. Employment (full or parttime); Significant; NanoString Technologies. M.D. Bailey: A. Employment (full or part-time); Significant; NanoString Technologies. C.R. Merritt: A. Employment (full or part-time); Significant; NanoString Technologies. M. Hoang: A. Employment (full or part-time); Significant; NanoString Technologies. S. Warren: A. Employment (full or part-time); Significant; NanoString Technologies. J.M. Beechem: A. Employment (full or part-time); Significant; NanoString Technologies.

\section{P03.06 PATTERN OF KI67 ${ }^{+}$EXPANDING CD8 ${ }^{+}$CYTOTOXIC T CELLS IN HEALTHY TISSUES, INFLAMMATION AND THE CANCER MICROENVIRONMENT}

K Möller, M Lennartz, R Abu-Hashem, NC Blessin, T Mandelkow, E Bady, C Hube-Magg*, R Simon, G Sauter, C Fraune, TS Clauditz, F Büscheck, AM Luebke. University Medical Center Hamburg-Eppendorf, Hamburg, Germany

\subsection{6/jitc-2020-ITOC7.46}

Background Expansion of $\mathrm{CD}^{+}$cytotoxic $\mathrm{T}$ lymphocytes is a prerequisite for anti-cancer immune activity. In the era of immune checkpoint therapy, profound knowledge of the dynamics of $\mathrm{CD}^{+}$has regained considerable interest. However, systematically acquired data on $\mathrm{CD}^{+}$proliferation in large sets of normal and diseased tissues are sparse.

Materials and Methods Here, we applied multiplex fluorescence immunohistochemistry to conventional large sections and tissue microarrays in order to quantitate $\mathrm{Ki} 67^{+} \mathrm{CD} 8^{+}$cells in $>20$ different compartments of normal lymphoid tissues, 7 types of inflammatory diseases and 785 cancers.

Results In most normal lymphoid tissues (tonsil, lymph node, thymus, Peyer's patches, spleen, colon, appendix) the percentage of $\mathrm{Ki} 67^{+} \mathrm{CD} 8^{+}$cells typically did not exceed $3 \%$. The percentage of $\mathrm{Ki} 67^{+} \mathrm{CD} 8{ }^{+}$cells was markedly higher $(45 \%)$ in the immune-active cortex of the thymus, however. In inflammatory conditions (including Hashimoto thyroiditis, Lichen sclerosus of the penis, sarcoidosis, sialadenitis, IgG4 pancreatitis, Crohn's disease and eczema), the percentage of $\mathrm{Ki}^{+} 7^{+} \mathrm{CD}^{+}$cells was much more variable and often sharply higher than in normal tissues. It ranged from $0.5 \%$ in one patient with sialadenitis to $19 \%$ in the intraepithelial compartment of Crohn's disease. In 765 colorectal cancers, the fraction of $\mathrm{Ki} 67$ positive $\mathrm{CD} 8^{+}$cytotoxic $\mathrm{T}$ cells ranged from 0 to $100 \%$ (mean: $20.6 \%$ ). A high fraction of $\mathrm{Ki}^{2} 7^{+} \mathrm{CD}^{+}$cells was significantly associated with microsatellite instability $(\mathrm{p}<0.0001)$, low pT stage $(\mathrm{p}<0.0001)$ and absence of nodal metastases $(p=0.0005)$.

Conclusions In summary, our data show a variable increase of the fraction of proliferating $\mathrm{CD}^{+} \mathrm{T}$ cells in cancers and in 